\title{
"Playing it SAFE in the NICU" SAFE-R: a targeted diagnostic ultrasound protocol for the suddenly decompensating infant in the NICU
}

\author{
Nadya Yousef ${ }^{1} \cdot$ Yogen Singh ${ }^{2,3} \cdot$ Daniele De Luca ${ }^{1,4}$
}

Received: 25 February 2021 / Revised: 26 May 2021 / Accepted: 24 June 2021 / Published online: 5 July 2021

(c) The Author(s), under exclusive licence to Springer-Verlag GmbH Germany, part of Springer Nature 2021

\begin{abstract}
Rapid diagnosis of sudden, unexpected, and potentially fatal complications in the neonatal intensive care unit (NICU) is essential for the initiation of prompt and life-saving management. Point-of-care ultrasound (POCUS) protocols are widely used in adult emergency situations to diagnose and guide treatment, but none has been specifically developed for the neonate. We propose a targeted diagnostic ultrasound protocol for the suddenly decompensating infant in the NICU for rapid screening for the most common life-threatening complications needing immediate attention. We integrated current knowledge on the use of POCUS for diagnosis of the most critical neonatal complications into the "SAFE-R protocol" (Sonographic Assessment of liFethreatening Emergencies - Revised). The ultrasound algorithm was evaluated at the bedside for suitability and ease of use. Main features of SAFE-R are the use of standardized ultrasound points and a simple one-probe rule-in/rule-out approach. The flowchart is designed by order of urgency and priority is given to treatable causes. Hence, ruling out cardiac tamponade is the first step in the decision tree, followed by pneumothorax, pleural effusion, then acute critical aortic occlusion, acute abdominal complications, and severe intraventricular hemorrhage.

Conclusion: SAFE-R is the first ultrasound algorithm specifically conceived for use in the NICU to screen for the most common urgent neonatal complications leading to sudden deterioration, thereby providing critical information within minutes. The simplified and rapid approach is designed for the neonatologist and is easy to learn and quick to perform.

\section{What is Known:}

- The fields of neonatal and pediatric critical care are undergoing a transformation with the adoption of POCUS and the recent publication of the first international guidelines on POCUS for critically ill children and neonates.

- Targeted emergency ultrasound protocols are widely used in adult emergency and critical care medicine, but specific and adapted ultrasound algorithms are lacking for the pediatric and neonatal population.

\section{What is New:}

- We propose the first targeted ultrasound protocol specifically designed for the suddenly decompensating infant in the NICU for rapid screening of the most common life-threatening complications needing immediate attention.

- The SAFE-R ultrasound algorithm integrates current knowledge on ultrasound diagnosis of the most critical neonatal complications into a simple and easy-to-perform emergency scanning protocol aimed to guide initial management and resuscitation efforts.
\end{abstract}

Keywords Point-of-care ultrasound $\cdot$ Neonatal critical care $\cdot$ Resuscitation $\cdot$ Ultrasound protocol

Communicated by Piet Leroy

Nadya Yousef

nadya.yousef@aphp.fr

1 Division of Pediatrics and Neonatal Critical Care, "A. Béclère" Medical Center, Paris Saclay University Hospitals, APHP, 157 rue de la Porte de Trivaux, 92140 Clamart, Paris, France

2 Department of Pediatrics - Neonatology and Pediatric Cardiology, Cambridge University Hospitals NHS

Foundation Trust, Cambridge, UK
3 Loma Linda University School of Medicine, Loma Linda, CA, USA

4 Physiopathology and Therapeutic Innovation Unit-INSERM U999, Paris Saclay University, Paris, France 


\section{Abbreviations \\ CHD Congenital heart disease \\ NICU Neonatal intensive care unit \\ POCUS Point-of-care ultrasound \\ SAFE Sonographic Assessment of liFe-threatening Emergencies}

SAFE-R Sonographic Assessment of liFe-threatening Emergencies - Revised

\section{Background}

The use of point-of-care ultrasound (POCUS) has rapidly increased in the past years, especially in critical care and emergency medicine. Adult critical care providers have pioneered the field with publication of formal guidelines and elaboration of targeted ultrasound protocols that are widely used in many situations, e.g., dyspnea [1], shock [2], and trauma [3].

Although the use of POCUS has been slower to gain momentum in pediatrics and neonatology, the past years have seen significant expansion in its use, particularly in pediatric emergency medicine [4] and in neonatal critical care $[5,6]$.

The recent publication of the first international evidencebased guidelines for the use of POCUS in neonatal and pediatric critical care provides a first framework to guide the use of POCUS in critically ill children and newborns [7]. However, formal diagnostic integrated ultrasound protocols for use in emergency situations, as in adult medicine, are still lacking.

With the growth of POCUS in neonatal intensive care, there is a need for specific ultrasound protocols adapted to the clinical context, and to the age and size of the patient. Rapid diagnosis of unexpected and potentially fatal complications in the neonatal intensive care unit (NICU) is essential to prompt life-saving management in the newborn. Our objective was to propose a simple and easy-to-use ultrasound protocol for the suddenly deteriorating infant in the NICU to screen for the most common life-threatening complications and to guide initial management.

\section{Materials and methods}

The proposed ultrasound protocol was developed in an academic tertiary level NICU where POCUS is routinely performed as a first line imaging technique for patients with respiratory and/or hemodynamic compromise and in the case of sudden clinical deterioration.

The protocol is specifically intended for use in previously stable infants in the NICU who present with acute decompensation with bradycardia and/or prolonged significant desaturation needing resuscitation, or rapidly increasing oxygen requirements, to maintain stable saturation levels, when there is no apparent explanation, e.g., technical difficulties such as an occluded or dislodged ETT and ventilator dysfunction.

The first step of the proposed SAFE-R protocol is the SAFE protocol (Sonographic Assessment of liFe-threatening Emergencies) [6] which integrates current knowledge on the POCUS detection of the most critical neonatal complications (cardiac tamponade, tension pneumothorax, and large pleural effusions) into a simple POCUS algorithm. SAFE was assessed at the bedside for suitability and ease of use [8].

Operators underwent basic training consisting of a 2-h theoretical lung ultrasound course followed by hands-on training in the NICU. The SAFE protocol was then evaluated prospectively in a bi-center, international, observational study in two tertiary level NICUs over 2 months by operators with different levels of experience defined as novices (experience $<3$ months) and experienced sonographers ( $>6$ months of ultrasound experience). All operators, regardless of their level of experience, were able to quickly screen for cardiac tamponade, pneumothorax and pleural effusion. Mean time to complete the SAFE proto-

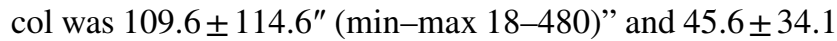
(min-max 20-180") for novice and experienced ultrasonographers, respectively $(\mathrm{p}=0.014)$ [Unpublished results. Foligno S, Meau-Petit V, Tusor N, Paltrinieri A, De Luca D, Yousef N. NICU evaluation of the SAFE ultrasound algorithm (Sonographic Assessment for liFe-threatening Emergencies) by differently experienced operators. 2nd LAUNCH conference, Paris, Feb 13-14, 2020].

The SAFE protocol was also evaluated independently in a tertiary referral hospital over 3 years [Unpublished results. Ibarra-Rios D. Use of the sonographic algorithm for life threatening emergencies in a tertiary level referral hospital in Mexico City. Case series over 3 years. 2nd launch conference, March 12, 2021]. The protocol was performed on 12 'crashing' patients before conventional radiography was performed (5 patients by an attending physician with expertise, 5 patients by fellows supervised by attending physician, and 2 patients exclusively by trained fellows), and a total of ten emergency procedures were performed as a result ( 3 pleural tubes insertions, 2 pleural tubes repositioned, 2 needle aspirations for pneumothorax, 2 needle aspirations for pleural effusion and one tamponade drainage). One hundred percent of patients survived the event.

We here intended to revise the SAFE protocol and extend it to include extra-thoracic causes of acute decompensation in the NICU; acute critical aortic occlusion, acute abdominal complications, and severe 
intraventricular hemorrhage were integrated into SAFE-R (Sonographic Assessment of liFe-threatening Emergencies Revised).

Ethical approval was not sought since the presented research did not involve patients.

\section{Results}

Main features of SAFE-R are standardized ultrasound points and a simple one-probe, rule-in/rule-out approach. The protocol is designed for the average neonatologist and requires minimal training to perform.

As shown in Fig. 1, the SAFE-R decision tree is designed by order of urgency. Hence, as a preliminary step, cardiac contractility is rapidly evaluated using an "eyeball" method [7], and expert help is called for in case of severe myocardial dysfunction. Then, cardiac tamponade, which is a rare condition, is ruled out as the first step in the decision tree since it may be fatal in the absence of prompt intervention, followed by tension pneumothorax, pleural effusion, acute critical aortic occlusion, acute abdominal complications, and severe intraventricular hemorrhage.

Priority is given to treatable causes of decompensation, and the order of the standardized points in the decision tree was chosen to allow for a logical and easy movement of the ultrasound probe from the thorax, to the abdominal aorta, the iliac fossae, and then the head (Fig. 2).

Pulsatility of the abdominal aorta is assessed by moving the ultrasound to the subcostal window. In the absence of aortic pulsatility, critical aortic occlusion must be suspected and urgent expert help must be sought. The next step is to place the probe in the iliac fossae where signs of abundant intra-abdominal fluid would orient suspicion to an acute abdominal cause of decompensation and warrant targeted workup and management. Finally, as a last step, the probe is placed on the fontanelle to screen for severe intraventricular hemorrhage. Although this complication is non-treatable, supportive therapy of e.g., associated anemia may be necessary to achieve patient stability. SAFER is not designed for detection of congenital heart diseases, and it should not be used to rule out congenital heart disease. The risk of undiagnosed

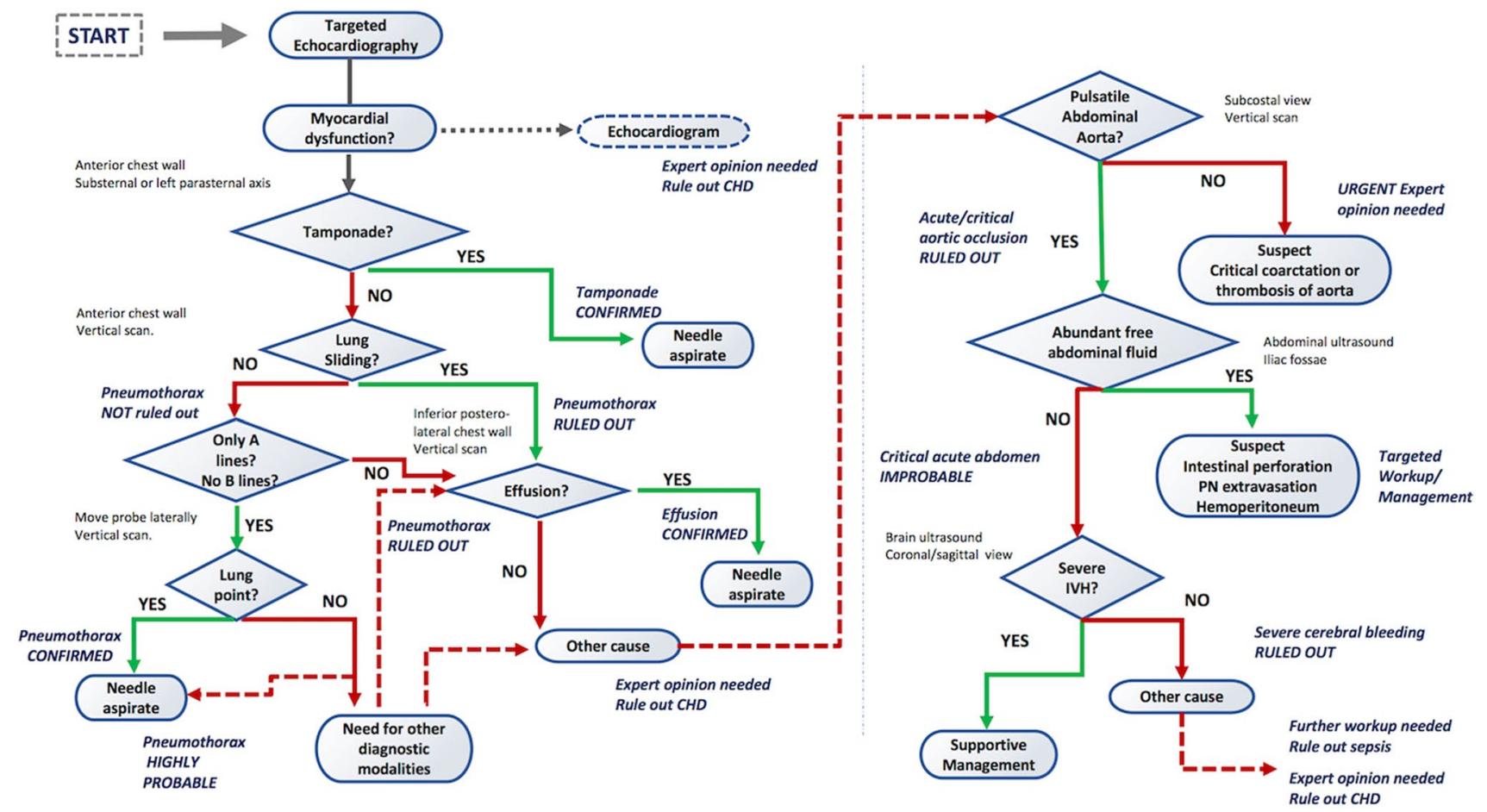

Fig. 1 The SAFE-R protocol flowchart. The step-by-step ultrasound protocol uses a single probe at standardized SAFE points with a simple rule in/rule out approach. The decision tree is designed by urgency, and the order of the standardized points in the decision tree allows for a logical and easy use of the ultrasound probe from the thorax, to the abdominal aorta, the iliac fossae, and then the head. Starting at the upper left with the most urgent intra-thoracic causes the following: cardiac tamponade, tension pneumothorax and pleural effusion, the protocol includes extra-thoracic causes at the right hand of the figure with critical aortic occlusion, abdominal complication, and severe intraventricular hemorrhage. An important reminder of the possibility of congenital heart disease (CHD) needing a full echocardiogram by an expert is indicated on both sides of the panel, since for the neonatal population, this remains an important differential diagnosis. The color version uses green and red arrows to signify positive or negative ultrasound findings, with the conclusion and comments in blue 
critical congenital heart disease is always present in the neonatal population, and therefore, it should always remain as a differential diagnosis needing expert attention.

\section{Discussion/conclusion}

The SAFE-R protocol is the first specific formal ultrasound algorithm adapted for use in the NICU. The NICU neonate is a critically ill infant, often preterm and usually needing respiratory and/or hemodynamic support, generally for several weeks. Patients are exposed to potentially serious complications related to their underlying disease and/or extremely low gestational age, in addition to those related to invasive procedures, e.g., central lines and mechanical ventilation. Current diagnostic modalities are mainly limited to chest X-rays, because of the limitations related to the use of CT scans and other imaging modalities. POCUS offers the advantage of being quick to perform at the bedside, repeatable, non-invasive, and offers a high diagnostic

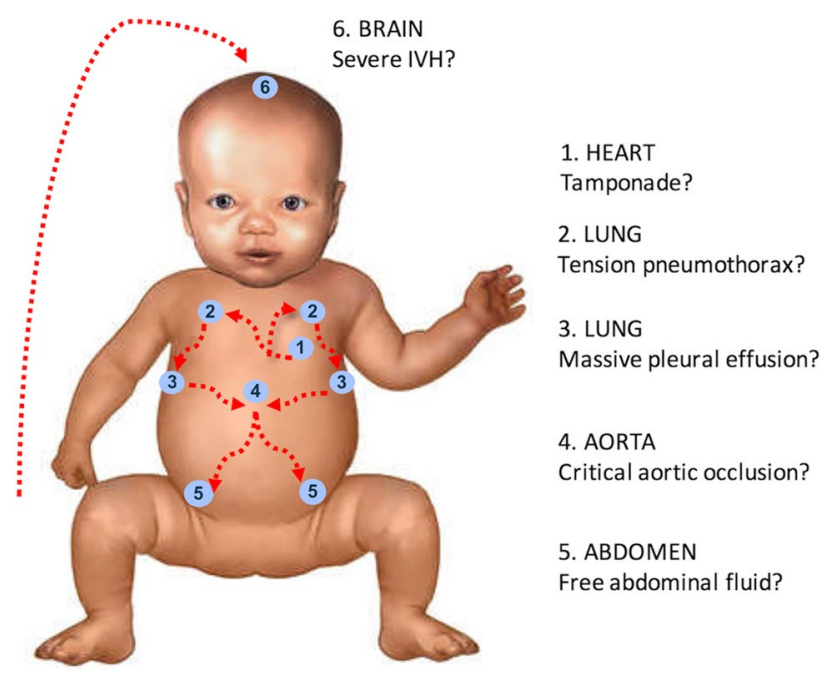

Fig. 2 A SAFE baby: The SAFE points in the SAFE-R protocol. (C) 2012-2020 Nucleus Medical Media. All rights reserved. With permission. The probe is first placed on the chest at the left parasternal or substernal axis (point 1) or subcostally. As preliminary step, cardiac contractility is rapidly evaluated using an "eyeball" method, and expert help is called for in case of myocardial dysfunction. The protocol starts with assessment for tamponade, then the probe is moved to the anterior chest wall bilaterally to assess for tension pneumothorax (point 2), and then to the inferior posterolateral chest wall bilaterally (point 3) to assess for pleural effusion. The probe is then moved to the substernal notch (point 4) to assess for the pulsatility of the abdominal aorta as screening of critical aortic occlusion and then to the iliac fossae (point 5) to assess for abundant intra-abdominal free fluid, before being placed on the anterior fontanel (point 6) to evaluate for severe intracranial hemorrhage. The order of the standardized points in the decision tree was chosen to allow for a logical and easy use of the ultrasound probe from the thorax, to the abdominal aorta, the iliac fossae, and then the head performance compared with the chest X-ray for diagnosis of the most life-threatening emergencies in the NICU [4-6].

A main challenge to implementation of POCUS in general is the need for adapted training and effort to achieve competency and skills. However, for the targeted POCUS, studies show that short focused courses improve ranging from a few hours to a few days are sufficient to achieve basic POCUS competency $[9,10]$ and for implementation into daily practice even for novices. Mazmanyan P et al. [11] were able to introduce and implement lung ultrasound into daily practice in a middle-income country after a short lung ultrasound training course.

The proposed SAFE-R protocol relies on simple ultrasound signs that need short training and little experience to use. It is designed for use in the case of acute decompensation in the NICU in previously stable infants once more common causes (e.g., ETT occlusion or dislodgement, ventilator dysfunction) have been addressed. In these previously stable (or relatively stable) babies presenting with acute severe deterioration cardiac or respiratory arrest or near-arrest, or needing increased respiratory support to maintain stable saturation values, the initiation of targeted intervention may be highly time sensitive.

Bedside evaluation of the SAFE protocol, which is the first step in SAFE-R, shows that basic training is sufficient to allow operators, regardless of prior ultrasound experience, to quickly screen for cardiac tamponade, pneumothorax, and pleural effusion (Foligno S et al., LAUNCH 2020) and that it can be successfully used by residents on-call in emergency situations [Ibarra-Rio D, 3rd LAUNCH 2021].

SAFE-R allows for a rapid screening of urgent complications of central lines needing rapid intervention such as cardiac tamponade, thoracic and abdominal extravasation of parenteral fluid, as well as tension pneumothorax, which may complicate ventilation or an underlying respiratory pathology in the newborn. The screening of the abdominal aorta may help in suspecting critical duct-dependent congenital heart disease (CHD) as a cause of acute decompensation and permit initiation of life-saving treatment while awaiting expert evaluation by a full echocardiogram, which, unfortunately, may not always be immediately available. Assessment of the abdomen can orient suspicion towards an abdominal complication such as necrotizing enterocolitis and/or gut perforation in case of abundant free intraperitoneal fluid. Large abdominal effusions due to less common causes may also be detected (e.g., bleeding, lymphatic malformations). Severe intraventricular hemorrhage is a feared complication in preterm neonates and may need supportive treatment to correct anemia and/or acute hypovolemia.

A main feature of SAFE- $R$ is the use of a single ultrasound probe. Gomond-Le Goff et al. showed good interrater agreement and reliability, irrespective of the probe 
and operator expertise, for neonatal lung ultrasound [12]. Although, for SAFE-R, theoretically, any ultrasound probe may be used, a separate specific study to determine the optimal probe for this purpose is necessary.

The SAFE-R protocol is designed to help the neonatologist in the decision-making process when faced with a suddenly and severely decompensating infant in the NICU. It aims to rapidly screen for the most common life-threatening complications needing immediate attention, thereby allowing the clinician to quickly initiate treatment or, in the case of a negative screen, to promptly assess for other neonatal conditions such as sepsis, or decompensation of metabolic or endocrine disorders, or CHD needing urgent comprehensive echocardiography. Since it needs minimal training to perform, SAFE-R may help guide junior clinicians in the management of patients while awaiting more expert help, thereby saving valuable time in emergencies. SAFE-R may also be useful for more POCUS-experienced clinicians who may use SAFE-R and/or combine it with a full echocardiogram or a more extensive POCUS evaluation.

The purpose of our study was to propose an integrated ultrasound protocol for use in a complex clinical situation as a step towards the integration of POCUS into emergency situations in the NICU. A field validation of the proposed protocol is necessary before it can be implemented into daily practice. A multi-center study is planned to evaluate SAFE$\mathrm{R}$ at the bedside with operators with variable levels of experience. It is also our intention to submit SAFER protocol to a Delphi consensus to the next edition of the PICU/NICU POCUS guidelines issued by the European Society for Pediatric and Neonatal Intensive Care [7].

In spite of numerous barriers to its implementation into daily practice, POCUS is transforming the field of pediatric and neonatal critical care. The proposed SAFE-R is the first ultrasound protocol specifically designed for use in the NICU to screen for the most common urgent neonatal complications leading to sudden deterioration, thereby providing critical information within minutes. The simplified and rapid approach is designed for the neonatologist and is easy to learn and quick to perform and hopefully a step towards improving the quality of care to our most vulnerable patients.

Acknowledgements The authors are grateful to Dr. Virginie Meau-Petit for her valuable suggestions and to Dr. Shivani Shankar-Aguilera for her constructive comments on the manuscript.

Authors' contributions Dr. Yousef conceived and designed the protocol, performed the literature search, wrote the first manuscript draft, and prepared the figures. Dr. Singh helped with design of the protocol and preparation of the figures. A/Prof. De Luca helped with protocol design and conception, and with literature search. All authors reviewed the manuscript for important intellectual content, agreed to be accountable for all aspects of the work, and approved the final manuscript version to be submitted.

\section{Declarations}

Competing interests Dr. Yousef and A/Prof. De Luca have received grants and honoraria from Chiesi Pharmaceuticals. Dr. Yousef and A/ Prof. De Luca have served as consultants and lecturers for Chiesi Pharmaceuticals. A/Prof. De Luca has been a member of the advisory board for Chiesi Pharmaceuticals. A/Prof. De Luca has received grants from and been an advisory board member of AbbVie and served as consultant and lecturer for Airway Therapeutics and AbbVie. These companies have had no role in the design, preparation, review, or approval of the manuscript or the decision to submit it for publication. Dr. Singh has served as a lecturer and received honorarium from AbbVie, Orphan Europe / Rorcordati Rare Diseases UK. He is Chair of Data and Safety Monitoring Board (DSMB) for a research project funded by GE. These companies have had no role in the design, preparation, review, or approval of the manuscript or the decision to submit it for publication.

\section{References}

1. Lichtenstein D, Meziere GA (2008) Relevance of lung ultrasound in the diagnosis of acute respiratory failure: the BLUE protocol. Chest 134:117-125. https://doi.org/10.1378/chest.07-2800

2. Atkinson P, Bowra J, Milne J, Lewis D, Lambert M, Jarman B, Noble VE, Lamprecht H, Harris T, Connolly J, members of the International Federation of Emergency Medicine Sonography in Hypotension and Cardiac Arrest working group (2017) International Federation for Emergency Medicine Consensus Statement: sonography in hypotension and cardiac arrest (SHoC): an international consensus on the use of point of care ultrasound for undifferentiated hypotension and during cardiac arrest. CJEM 19:459-470. https://doi.org/10.1017/cem.2016.394

3. Scalea TM, Rodgriguez A, Chiu WC, Brenneman FD, Fallon WF Jr, Kato K, McKenny MG, Nerlich ML, Ochsner YH (1999) Focused Assessment with Sonography for Trauma (FAST): results from an international consensus statement. J Trauma 46:466-472. https://doi.org/10.1097/00005373-199903000-00022

4. Marin JR, Abo AM, Arroyo AC et al (2016) Pediatric emergency medicine point-of-care: summary of the evidence. Crit Ultrasound J 8:16. https://doi.org/10.1186/s13089-016-0049-5

5. Miller LE, Stoller JZ, Fraga MV (2020) Point of care ultrasound in the neonatal ICU. Curr Opin Pediatr 32:216-227. https://doi. org/10.1097/MOP.0000000000000863

6. Raimondi F, Yousef N, Migliaro F, Capasso L, De Luca D (2018) Point-of-care lung ultrasound in neonatology: classification into descriptive and functional applications. Pediatr Res 20:1-8. https://doi.org/10.1038/s41390-018-0114-9

7. Singh Y, Tissot C, Fraga MV et al (2020) International evidencebased guidelines on Point of Care Ultrasound (POCUS) for critically ill neonates and children issued by the POCUS Working Group of the European Society of Paediatric and Neonatal Intensive Care (ESPNIC). Crit Care 24;24(1):65. https://doi.org/10. 1186/s13054-020-2787-9

8. Yousef N, Shankar-Aguilera S, De Luca D (2017) The SAFE protocol: a sonographic algorithm for life-threatening emergencies in the neonatal intensive care unit. J Pediatr Neonatal Individualized Med 2017; 6 (2-:e060236): 94 [abstract 107]. https://doi.org/10. 7363/060236

9. Tuvali O, Sadeh R, Kobal S, Yarza S, Golan Y, Fuchs L (2020) The long-term effect of short point of care ultrasound course on physicians' daily practice. PLoS One 15(11):e0242084. https:// doi.org/10.1371/journal.pone.0242084 
10. Dinh VA, Giri PC, Rathinavel I, Nguyen E, Hecht D, Dorotta I, Nguyen HB, Chrissian AA (2015) Impact of a 2-Day Critical Care Ultrasound Course during Fellowship Training: A Pilot Study. Crit Care Res Pract 2015:675041. https://doi.org/10.1155/2015/675041

11. Mazmanyan P, Kerobyan V, Shankar-Aguilera S, Yousef N, De Luca D (2020) Introduction of point-of-care neonatal lung ultrasound in a developing country. Eur J Pediatr 179(7):11311137. https://doi.org/10.1007/s00431-020-03603-w
12. Gomond-Le Goff C, Vivalda L, Foligno S, Loi B, Yousef N, De Luca D (2020) Effect of different probes and expertise on the interpretation reliability of point-of-care lung ultrasound. 157(4):924-931. https://doi.org/10.1016/j.chest.2019.11.013

Publisher's Note Springer Nature remains neutral with regard to jurisdictional claims in published maps and institutional affiliations. 